\title{
On 1-absorbing $\delta$-primary ideals
}

\author{
Abdelhaq El Khalfi, Najib Mahdou, Ünsal Tekir and Suat Koç
}

\begin{abstract}
Let $R$ be a commutative ring with nonzero identity. Let $\mathcal{J}(R)$ be the set of all ideals of $R$ and let $\delta: \mathcal{J}(R) \longrightarrow \mathcal{J}(R)$ be a function. Then $\delta$ is called an expansion function of ideals of $R$ if whenever $L, I, J$ are ideals of $R$ with $J \subseteq I$, we have $L \subseteq \delta(L)$ and $\delta(J) \subseteq \delta(I)$. Let $\delta$ be an expansion function of ideals of $R$. In this paper, we introduce and investigate a new class of ideals that is closely related to the class of $\delta$-primary ideals. A proper ideal $I$ of $R$ is said to be a 1-absorbing $\delta$-primary ideal if whenever nonunit elements $a, b, c \in R$ and $a b c \in I$, then $a b \in I$ or $c \in \delta(I)$. Moreover, we give some basic properties of this class of ideals and we study the 1-absorbing $\delta$-primary ideals of the localization of rings, the direct product of rings and the trivial ring extensions.
\end{abstract}

\section{Introduction}

Throughout this paper, all rings are assumed to be commutative with nonzero identity and all modules are nonzero unital. If $R$ is a ring, then $\sqrt{I}$ denotes the radical of an ideal $I$ of $R$, in the sense of [12, page 17]. Let also $\operatorname{Spec}(R)$ denotes the set of all prime ideals of $R$.

The prime ideal, which is an important subject of ideal theory, has been widely studied by various authors. Among the many recent generalizations of the notion of prime ideals in the literature, we find the following, due to Badawi [2]. A proper ideal $I$ of a ring $R$ is said to be a 2 -absorbing ideal if whenever

Key Words: 1-absorbing prime ideal, 1-absorbing $\delta$-primary ideal, $\delta$-primary ideal, trivial ring extension.

2010 Mathematics Subject Classification: 13A99, 13C13.

Received: 09.03.2021

Accepted: 30.04 .2021 
$a, b, c \in R$ and $a b c \in I$, then $a b \in I$ or $a c \in I$ or $b c \in I$. In this case $\sqrt{I}=P$ is a prime ideal with $P^{2} \subseteq I$ or $\sqrt{I}=P_{1} \cap P_{2}$ where $P_{1}, P_{2}$ are incomparable prime ideals with $\sqrt{I}^{2} \subseteq I$, cf. [2, Theorem 2.4]. Recently, Badawi and Yetkin [4] consider a new class of ideals called the class of 1-absorbing primary ideals. A proper ideal $I$ of a ring $R$ is called a 1-absorbing primary ideal of $R$ if whenever nonunit elements $a, b, c \in R$ and $a b c \in I$, then $a b \in I$ or $c \in \sqrt{I}$. In [14], A. Yassine et. al introduced the concept of 1-absorbing prime ideals which is a generalization of prime ideals. A proper ideal $I$ of a $\operatorname{ring} R$ is a 1-absorbing prime ideal if whenever we take nonunit elements $a, b, c \in R$ with $a b c \in I$, then $a b \in I$ or $c \in I$. In this case $\sqrt{I}=P$ is a prime ideal, cf. [14, Theorem 2.3]. If $R$ is a ring in which exists a 1-absorbing prime ideal that is not prime, then $R$ is a local ring, that is a ring with one maximal ideal.

Let $\mathcal{J}(R)$ be the set of all ideals of a ring $R$. Zhao [15] introduced the concept of expansion of ideals of $R$. We recall from [15] that a function $\delta$ : $\mathcal{J}(R) \longrightarrow \mathcal{J}(R)$ is called an expansion function of ideals of $R$ if whenever $L, I, J$ are ideals of $R$ with $J \subseteq I$, we have $L \subseteq \delta(L)$ and $\delta(J) \subseteq \delta(I)$. Note that there are explanatory examples of expansion functions included in [15, Example 1.2] and [3, Example 1]. In addition, recall from [15] that a proper ideal $I$ of $R$ is said to be a $\delta$-primary ideal of $R$ if whenever $a, b \in R$ with $a b \in I$, we have $a \in I$ or $b \in \delta(I)$, where $\delta$ is an expansion function of ideals of $R$. Also, recall from [5] that a proper ideal $I$ of $R$ is called a $\delta$-semiprimary ideal of $R$ if $a b \in I$ implies $a \in \delta(I)$ or $b \in \delta(I)$. In this paper, we introduce and investigate a new concept of ideals that is closely related to the class of $\delta$-primary ideals. A proper ideal $I$ of $R$ is said to be a 1-absorbing $\delta$-primary ideal if whenever nonunit elements $a, b, c \in R$ and $a b c \in I$, then $a b \in I$ or $c \in \delta(I)$. For example, let $\delta_{1}: \mathcal{J}(R) \longrightarrow \mathcal{J}(R)$ such that $\delta_{1}(I)=\sqrt{I}$ for each ideal $I$ of $R$. Then $\delta_{1}$ is an expansion function of ideals of $R$, and hence a proper ideal $I$ of $R$ is a 1absorbing $\delta_{1}$-primary ideal of $R$ if and only if $I$ is a 1-absorbing primary ideal of $R$. Among many results in this paper are given to disclose the relations between this new class and others that already exist. The reader may find it helpful to keep in mind the implications noted in the following figure.

prime ideal $\longrightarrow$ 1-absorbing prime ideal $\longrightarrow 1$-absorbing $\delta$-primary ideal

Among other things, we give an example of 1 -absorbing $\delta$-primary ideal that is not 1-absorbing prime ideal (Example 2.3). Also, we show (Theorem 2.6 ) that if a ring $R$ admits a 1-absorbing $\delta$-primary ideal of $R$ that is not a $\delta$-primary ideal, then $R$ is a local ring. Moreover, we prove that if $R$ is a chained ring with maximal ideal $M$, then the only 1-absorbing $\delta$-primary 
ideals of $R$ are $M^{2}$ and the $\delta$-primary ideals of $R$ (Theorem 2.11). Finally, we give an idea about 1 -absorbing $\delta$-primary ideals of the localization of rings, the direct product of rings and the trivial ring extensions.

\section{Main Results}

We start this section by the following definition.

Definition 2.1. A proper ideal $I$ of a ring $R$ is called a 1 -absorbing $\delta$-primary ideal if whenever abc $\in I$ for some nonunit elements $a, b, c \in R$, then $a b \in I$ or $c \in \delta(I)$.

Remark 2.2. Let $R$ be a ring, $I$ a proper ideal of $R$ and $\delta$ be an expansion function of $\mathcal{J}(\mathcal{R})$.

(1) If $\delta(I)=I$, then $I$ is a 1-absorbing $\delta$-primary ideal of $R$ if and only if it is a 1-absorbing prime ideal.

(2) If $\delta(I)=\sqrt{I}$, then $I$ is a 1-absorbing $\delta$-primary ideal of $R$ if and only if it is a 1-absorbing primary ideal.

(3) Every 1-absorbing prime ideal is a 1-absorbing $\delta$-primary ideal.

(4) Every $\delta$-primary ideal is a 1-absorbing $\delta$-primary ideal.

(5) Let $\gamma$ be an expansion function of $\mathcal{J}(\mathcal{R})$ such that $\delta(I) \subseteq \gamma(I)$. If I is a 1-absorbing $\delta$-primary ideal of $R$, then $I$ is a 1-absorbing $\gamma$-primary ideal of $R$.

Next, we give an example of a 1-absorbing $\delta$-primary ideal that is not a 1-absorbing prime ideal.

Example 2.3. Let $R:=K\left[\left[X_{1}, X_{2}, X_{3}\right]\right]$ be a ring of formal power series where $K$ is a field. Consider the expansion function $\delta: \mathcal{J}(\mathcal{R}) \longrightarrow \mathcal{J}(\mathcal{R})$ defined by $\delta(I)=I+M$ where $M=\left(X_{1}, X_{2}, X_{3}\right)$ is the maximal ideal of $R$. Consider the ideal $I=\left(X_{1} X_{2} X_{3}\right)$ of $R$. Thus, $I$ is not a 1-absorbing prime ideal of $R$ since $X_{1} X_{2} X_{3} \in I$ but neither $X_{1} X_{2} \in I$ nor $X_{3} \in I$. Now, let $x, y, z$ be nonunit elements of $R$ such that $x y z \in I$. Clearly $I$ is a 1-absorbing $\delta$-primary because $z \in \delta(I)=M$.

Proposition 2.4. (i) Every 1-absorbing $\delta$-primary ideal is also a 2-absorbing $\delta$-primary ideal of $R$.

(ii) Let $I$ be a 1-absorbing $\delta$-primary ideal of $R$ and $\delta(I)$ be a radical ideal, that is, $\sqrt{\delta(I)}=\delta(I)$. Then $I$ is a $\delta$-semiprimary ideal of $R$. 
Proof. (i) Let $a b c \in I$ for some $a, b, c \in R$. If at least one of $a, b, c$ is a unit of $R$, then we are done. So assume that $a, b, c$ are nonunits of $R$. Since $I$ is a 1-absorbing $\delta$-primary ideal of $R$, we get $a b \in I$ or $c \in \delta(I)$, which implies that $a b \in I$ or $a c \in \delta(I)$ or $b c \in \delta(I)$. Therefore, $I$ is a 2-absorbing $\delta$-primary ideal of $R$.

(ii) Suppose that $a b \in I$ for some $a, b \in R$. Then we may assume that $a, b$ are nonunits. Thus $a^{2} b \in I$ implies that $a^{2} \in I$ or $b \in \delta(I)$. Then we have $a \in \sqrt{I} \subseteq \sqrt{\delta(I)}=\delta(I)$ or $b \in \delta(I)$. Hence, $I$ is a $\delta$-semiprimary ideal of $R$.

The converse of previous proposition (i) is not true in general. See the following example.

Example 2.5. (2-absorbing $\delta$-primary ideal that is not 1-absorbing $\delta$-primary ideal)

Let $R=\mathbb{Z}, I=p q \mathbb{Z}$, where $p \neq q$ are prime numbers, and $\delta(I)=I+p \mathbb{Z}$. Since $I$ is a 2-absorbing ideal, so is 2-absorbing $\delta$-primary. However, it is easy to see that $p p q \in I, p^{2} \notin I$ and $q \notin \delta(I)$. Thus, $I$ is not a 1-absorbing $\delta$-primary ideal of $R$.

In the next result, we show that if a ring $R$ admits a 1-absorbing $\delta$-primary ideal that is not a $\delta$-primary ideal, then $R$ is a local ring.

Theorem 2.6. Let $\delta$ be an ideal expansion. Suppose that a ring $R$ admits a 1 -absorbing $\delta$-primary ideal that is not a $\delta$-primary ideal. Then $R$ is a local ring.

Proof. Assume that $I$ is a 1 -absorbing $\delta$-primary ideal that is not a $\delta$-primary ideal of $R$. Hence there exist nonunit elements $a, b \in R$ such that $a b \in I$, $a \notin I$ and $b \notin \delta(I)$. Let $d$ be a nonunit element of $R$. As $d a b \in I, I$ is a 1 -absorbing $\delta$-primary ideal of $R$ and $b \notin \delta(I)$, we conclude that $d a \in I$. Let $c$ be a unit element of $R$. Suppose that $d+c$ is a nonunit element of $R$. Since $(d+c) a b \in I, I$ is a 1-absorbing $\delta$-primary ideal of $R$ and $b \notin \delta(I)$, we get that $(d+c) a=d a+c a \in I$. Since $d a \in I$, we conclude that $a \in I$, which gives a contradiction. Hence, $d+c$ is a unit element of $R$. Now, the result follows from [4, Lemma 1].

Next, we give a method to construct 1 -absorbing $\delta$-primary ideals that are not $\delta$-primary ideals.

Theorem 2.7. Let $R$ be a local ring with maximal ideal $M$ and $\delta$ be an ideal expansion. Let $x$ be a nonzero prime element of $R$ such that $\delta(x M) \subsetneq M$. If $x \in \delta(x M)$, then $x M$ is a 1-absorbing $\delta$-primary ideal of $R$ that is not a $\delta$-primary ideal of $R$. 
Proof. First, we will show that $x M$ is a 1-absorbing $\delta$-primary ideal of $R$. Assume that $a b c \in x M$ for some nonunit elements $a, b, c \in R$. If $a b \notin x M$, then $a \notin x R$ and $b \notin x R$, so $a b \notin x R$ because $x$ is a prime element of $R$. Moreover, the fact that $a b c \in x R$ and $a b \notin x R$ implies that $c \in x R \subseteq \delta(x M)$. Now, we prove that $x M$ is not a $\delta$-primary ideal of $R$. By hypothesis, we can pick an element $a \in M \backslash \delta(x M)$, hence $x a \in x M$. However, $x \notin x M$ since $x$ is an irreducible element of $R$ by [4, Lemma 2]. Which implies that $x M$ is not a $\delta$-primary ideal, this completes the proof.

Theorem 2.8. Suppose that $I$ is a 1-absorbing $\delta$-primary ideal of $R$ that is not a $\delta$-primary ideal of $R$. Then there exists an irreducible element $x \in$ $R$ and a nonunit element $y \in R$ such that $x y \in I$, but neither $x \in I$ nor $y \in \delta(I)$. Furthermore, if $a b \in I$ for some nonunit elements $a, b \in R$ such that neither $a \in I$ nor $b \in \delta(I)$, then $a$ is an irreducible element of $R$.

Proof. Suppose that $I$ is not a $\delta$-primary ideal of $R$. Hence neither $x \in I$ nor $y \in \delta(I)$ for some nonunit elements $x, y \in R$ with $x y \in I$. Assume on the contrary that $x$ is not an irreducible element of $R$. Then $x=c d$ for some nonunit elements $c, d \in R$. Since $x y=c d y \in I$ and $I$ is a 1 -absorbing $\delta$ primary ideal of $R$ and $y \notin \delta(I)$, we conclude that $c d=x \in I$, a contradiction. Hence, $x$ is an irreducible element of $R$.

Theorem 2.9. Let $I$ be a 1-absorbing $\delta$-primary ideal of a ring $R$ where $\delta$ is an ideal expansion and let $d \in R \backslash I$ be a nonunit element of $R$. Then $(I: d)=\{x \in R \mid d x \in I\}$ is a $\delta$-primary ideal of $R$. In particular, for every proper ideal $J$ of $R$ with $J \nsubseteq I,(I: J)$ is a $\delta$-primary ideal of $R$.

Proof. Suppose that $a b \in(I: d)$ for some elements $a, b \in R$. Without loss of generality, we may assume that $a$ and $b$ are nonunit elements of $R$. Suppose that $a \notin(I: d)$. Since $d a b \in I$ and $I$ is a 1-absorbing $\delta$-primary ideal of $R$, we conclude that $b \in \delta(I)$. So, $b \in \delta((I: d))$ and this completes the proof. The rest is similar.

Theorem 2.1. Let $\delta$ be an expansion function of $\mathcal{J}(\mathcal{R}), I$ a proper ideal of $R$ and $(I: x)=\left(I: x^{2}\right)$ for each $x \in R-\delta(I)$. If $I$ is an irreducible ideal, then $I$ is a 1-absorbing $\delta$-primary ideal of $R$.

Proof. Assume on the contrary that $I$ is not a 1 -absorbing $\delta$-primary ideal of $R$. Then there exist nonunit elements $a, b, c \in R$ with $a b c \in I$ and neither $a b \in I$ nor $c \in \delta(I)$. Consider $(I+R a b) \cap(I+R c)$. It is clear that $I \subseteq(I+R a b) \cap$ $(I+R c)$. Let $z \in(I+R a b) \cap(I+R c)$. Then there are $i_{1}, i_{2} \in I$ and $r_{1}, r_{2} \in R$ such that $z=i_{1}+r_{1} a b=i_{2}+r_{2} c$. Then $z c=i_{1} c+r_{1} a b c=i_{2} c+r_{2} c^{2} \in I$. Hence $r_{2} c^{2} \in I$; and so we have $r_{2} \in\left(I: c^{2}\right)=(I: c)$ by the assumption. So 
$z=i_{2}+r_{2} c \in I$. Thus we conclude that $I=(I+R a b) \cap(I+R c)$ which is a contradiction. Therefore, $I$ is a 1 -absorbing $\delta$-primary ideal of $R$.

Proposition 2.10. Let $R$ be a ring, $\delta$ an ideal expansion and $I$ be a proper ideal of $R$. If $I$ is a 1-absorbing $\delta$-primary ideal of $R$, then either $I$ is a $\delta$ semiprimary ideal of $R$ or $R$ is local, say with maximal ideal $M$, such that $M^{2} \subseteq I$.

Proof. If $R$ is not local, then Theorem 2.6 implies that $I$ is $\delta$-primary and so $I$ is a $\delta$-semiprimary ideal of $R$. Now, assume that $R$ is local with maximal ideal $M$ such that $I$ is not a $\delta$-semiprimary ideal of $R$. Since $I$ is proper, we infer that $I \subseteq M$. Observe that $\delta(I) \neq M$. Indeed, otherwise from Proposition 2.4 (ii), we conclude that $I$ is a $\delta$-semiprimary ideal which contradicts with our assumption. Moreover, there are $a, b \in M \backslash \delta(I)$ such that $a b \in I$. To prove that $M^{2} \subseteq I$, it suffices to show that $x y \in I$ for all $x, y \in M$. Let $x, y \in M$. Then $x y a b \in I$. Since $x y, a, b \in M, b \notin \delta(I)$ and $I$ is a 1 -absorbing $\delta$-primary ideal, we conclude that $x y a \in I$. Again, since $x, y, a \in M, a \notin \delta(I)$ and $I$ is a 1-absorbing $\delta$-primary ideal, we have that $x y \in I$.

Recall that a ring $R$ is a chained ring if the set of all ideals of $R$ is linearly ordered by inclusion. Moreover, $R$ is said to be an arithmetical ring if $R_{M}$ is a chained ring for each maximal ideal $M$ of $R$. We next determine the 1 -absorbing $\delta$-primary ideals of a chained ring.

Theorem 2.11. Let $R$ be a chained ring with maximal ideal $M, \delta$ an ideal expansion and $I$ be a proper ideal of $R$ such that $I \neq M^{2}$. Then $I$ is a 1 absorbing $\delta$-primary ideal of $R$ if and only if $I$ is a $\delta$-primary ideal of $R$.

Proof. We need only prove the "only if" assertion. Let $I$ be a 1-absorbing $\delta$-primary ideal. Thus, Proposition 2.10 gives that either $I$ is a $\delta$-semiprimary ideal of $R$ or $M^{2} \subseteq I$. First, assume that $I$ is a $\delta$-semiprimary ideal of $R$ and $a b \in I$ for some nonunit elements $a, b \in R$ such that $b \notin \delta(I)$. Hence, $a \in \delta(I)$. Now, since $R$ is a chained ring, we conclude that $a \in b R$ and thus $a=b r$ for some nonunit element $r \in R$. As $b r b \in I, b \notin \delta(I)$ and $I$ is a 1-absorbing $\delta$-primary ideal of $R$, we conclude that $a=b r \in I$. Which gives that $I$ is a $\delta$-primary ideal of $R$. Now, we suppose that $M^{2} \subseteq I$. We may assume that $M \neq I$. Thus, we can pick $a \in M \backslash I$ and $b \in I \backslash M^{2}$. Then $b \in a R$ since $R$ is a chained ring. So, $b=a r$ for some nonunit element $r \in R$ and thus $b \in M^{2}$, a contradiction. This completes the proof.

In view of Theorem 2.11, we have the following result.

Corollary 2.12. Let $R$ be an arithmetical ring with Jacobson radical $M$ and $I$ be a proper ideal of $R$ such that $I \neq M^{2}$. Then $I$ is a 1-absorbing $\delta$-primary ideal of $R$ if and only if $I$ is a $\delta$-primary ideal of $R$. 
Proof. Assume that $R$ is local with maximal ideal $M$. Since $R$ is an arithmetical ring, we conclude that $R=R_{M}$ is a chained ring and thus the claim follows from Theorem 2.11. In the remaining case, suppose that $R$ is not a local ring. Then the result follows by Theorem 2.6.

Proposition 2.13. Let $R$ be a local ring with principal maximal ideal $M, \delta$ an ideal expansion and $I$ be a proper ideal of $R$. Then $I$ is a 1-absorbing $\delta$ primary ideal of $R$ if and only if either $I$ is a $\delta$-primary ideal of $R$ or $M^{2} \subseteq I$. In addition, if $\sqrt{I} \subseteq \delta(I)$ then $I$ is a 1-absorbing $\delta$-primary ideal of $R$ if and only if $I$ is a $\delta$-primary ideal of $R$.

Proof. By Remark 2.2(4) and Proposition 2.10, we need only prove that if $I$ is a 1-absorbing $\delta$-primary ideal of $R$ which is a $\delta$-semiprimary ideal then $I$ is a $\delta$ primary ideal (along with the hypothesis that $R$ be a local ring with principal maximal ideal $M$ ). Also, we may assume that $\delta(I) \neq R$. Set $M=x R$ and let $a$ and $b$ be nonunit elements of $R$ such that $b \notin \delta(I)$ and $a b \in I$. Since $I$ is a $\delta$-semiprimary ideal of $R$, we get that $a \in \delta(I)$. Moreover, $a=r x$ for some $r \in R$. If $r$ is a unit element of $R$ then $M=\delta(I)$ and thus $I$ is a $\delta$-primary ideal. If $r$ is a nonunit element of $R$ then $r x b=a b \in I$. That implies $a=r x \in I$ since $I$ is a 1 -absorbing $\delta$-primary ideal. This completes the proof. The in addition statement is clear.

Proposition 2.14. Let $\left\{J_{i} \mid i \in D\right\}$ be a directed set of 1-absorbing $\delta$-primary ideals of $R$, where $\delta$ is an ideal expansion. Then the ideal $J=\cup_{i \in D} J_{i}$ is a 1 -absorbing $\delta$-primary ideal of $R$.

Proof. Let $a b c \in J$ for some nonunits $a, b, c \in R$, then $a b c \in J_{i}$ for some $i \in D$. Since $J_{i}$ is a 1-absorbing $\delta$-primary ideal of $R, a b \in J_{i}$ or $c \in \delta\left(J_{i}\right) \subseteq \delta(J)$. Hence, $J$ is a 1 -absorbing $\delta$-primary ideal of $R$.

Proposition 2.15. Let $I$ be a 1-absorbing $\delta$-primary ideal of $R$ such that $\sqrt{\delta(I)} \subseteq \delta(\sqrt{I})$, where $\delta$ is an ideal expansion. Then, $\sqrt{I}$ is a $\delta$-primary ideal of $R$.

Proof. Let $a b \in \sqrt{I}$ such that $a \notin \sqrt{I}$. Hence, there exists a positive integer $n$ such that $(a b)^{n} \in I$. So, $a^{m} a^{m} b^{n} \in I$ for some positive integer $m$. Since $I$ is a 1-absorbing $\delta$-primary ideal of $R$ and $a^{2 m} \notin I$, we conclude that $b^{n} \in \delta(I)$. That implies $b \in \sqrt{\delta(I)} \subseteq \delta(\sqrt{I})$ and so $\sqrt{I}$ is a $\delta$-primary ideal of $R$.

Proposition 2.16. Let $I$ be a proper ideal of a ring $R$ and $\delta$ be an ideal expansion such that $\delta(\delta(I))=\delta(I)$. Then the following statements are satisfied.

(1) If $I$ is a 1-absorbing $\delta$-primary ideal and $a, b$ are nonunit elements with $a b \notin I$, then $\delta(I: a b)=\delta(I)$.

(2) $\delta(I)$ is a 1-absorbing $\delta$-primary ideal of $R$ if and only if $\delta(I)$ is a 1 -absorbing prime ideal of $R$ 
Proof. (1) Let $I$ be a 1-absorbing $\delta$-primary ideal and $a b \notin I$. Note that $I \subseteq(I: a b)$ and so $\delta(I) \subseteq \delta(I: a b)$. Let $c \in(I: a b)$. Then $c \in \delta(I)$ since $a b c \in I$ and $a b \notin I$. Thus $(I: a b) \subseteq \delta(I)$. We get $\delta(I: a b) \subseteq \delta(\delta(I))=\delta(I)$. Hence we conclude the equality.

(2) By Remark 2.2(3), we need only prove the "only if" assertion. Let $a b c \in \delta(I)$ for some nonunit elements $a, b, c \in R$. Hence $a b \in \delta(I)$ or $c \in$ $\delta(\delta(I))=\delta(I)$. Thus $\delta(I)$ is a 1-absorbing prime ideal of $R$.

Proposition 2.17. Let $R$ be a ring, $I$ a proper ideal of $R$ and $\delta$ be an ideal expansion. Then $I$ is a 1-absorbing $\delta$-primary ideal if and only if whenever $I_{1} I_{2} I_{3} \subseteq I$ for some proper ideals $I_{1}, I_{2}$ and $I_{3}$ of $R$, then $I_{1} I_{2} \subseteq I$ or $I_{3} \subseteq \delta(I)$.

Proof. It suffices to prove the "if" assertion. Suppose that $I$ is a 1-absorbing $\delta$-primary ideal and let $I_{1}, I_{2}$ and $I_{3}$ be proper ideals of $R$ such that $I_{1} I_{2} I_{3} \subseteq I$ and $I_{3} \nsubseteq \delta(I)$. Thus $a b c \in I$ for every $a \in I_{1}, b \in I_{2}$ and $c \in I_{3} \backslash \delta(I)$. Since $I$ is a 1-absorbing $\delta$-primary ideal, we then have $I_{1} I_{2} \subseteq I$, as desired.

Recall from [15] that an ideal expansion $\delta$ is said to be intersection preserving if $\delta\left(I_{1} \cap I_{2} \cap \ldots \cap I_{n}\right)=\delta\left(I_{1}\right) \cap \delta\left(I_{2}\right) \cap \ldots \cap \delta\left(I_{n}\right)$ for any ideals $I_{1}, \ldots, I_{n}$ of $R$.

Proposition 2.18. Let $\delta$ be an intersection preserving ideal expansion. If $I_{1}, I_{2}, \ldots, I_{n}$ are 1-absorbing $\delta$-primary ideals of $R$, and $\delta\left(I_{i}\right)=P$ for some ideal $P$ of $R$ and all $i \in\{1,2, \ldots, n\}$, then $I_{1} \cap I_{2} \cap \ldots \cap I_{n}$ is a 1-absorbing $\delta$-primary ideal of $R$.

Proof. Let $a b c \in J=I_{1} \cap I_{2} \cap \ldots \cap I_{n}$ such that $a b \notin J$. Let $i \in\{1,2, \ldots, n\}$ such that $a b \notin I_{i}$. Since $a b c \in I_{i}$ and $I_{i}$ is a 1-absorbing $\delta$-primary ideal, we conclude that $c \in \delta\left(I_{i}\right)=\delta(J)$. Therefore, $J$ is a 1-absorbing $\delta$-primary ideal of $R$.

Proposition 2.19. Let $R$ be a ring and $\delta$ be an expansion function of $\mathcal{J}(R)$. Then the following statements are equivalent.

(1) Every proper principal ideal is a 1-absorbing $\delta$-primary ideal of $R$.

(2) Every proper ideal is a 1-absorbing $\delta$-primary ideal of $R$.

Proof. Assume that (1) holds and let $I$ be a proper ideal of $R$. Let $a, b, c$ be nonunit elements of $R$ such that $a b c \in I$. Hence $a b c \in a b c R$ which implies that $a b \in a b c R \subseteq I$ or $c \in \delta(a b c R) \subseteq \delta(I)$. Therefore $I$ is a 1-absorbing $\delta$-primary ideal of $R$. The converse is clear. 
An expansion function $\delta$ of $\mathcal{J}(R)$ is said to satisfy condition $(*)$ if $\delta(I) \neq R$ for each proper ideal $I$ of $R$. Note that the identity function and the radical operation are examples of expansion functions satisfying condition $(*)$.

Theorem 2.20. Let $R$ be a ring and $\delta$ an expansion function of $\mathcal{J}(R)$ satisfying condition $(*)$ and $\delta(\operatorname{Jac}(R))=\operatorname{Jac}(R)$. Suppose that $\delta(x I)=x \delta(I)$ for every proper ideal $I$ of $R$ and every $x \in R$. The following statements are equivalent.

(i) Every proper principal ideal is a 1-absorbing $\delta$-primary ideal of $R$.

(ii) Every proper ideal is a 1-absorbing $\delta$-primary ideal of $R$.

(iii) $R$ is local with $\operatorname{Jac}(R)^{2}=(0)$.

Proof. (i) $\Leftrightarrow$ (ii) Follows from Proposition 2.19.

$($ ii $) \Rightarrow$ (iii) Assume that every proper ideal is a 1-absorbing $\delta$-primary ideal $R$. Choose $x, y \in \operatorname{Jac}(R)$. Now, we will show that $x y=0$. If $x$ or $y$ is zero, then we are done. Assume that $x, y \neq 0$. Since $x^{2} y \in\left(x^{2} y\right)$ and $\left(x^{2} y\right)$ is a 1-absorbing $\delta$-primary ideal, we conclude that $x^{2} \in\left(x^{2} y\right)$ or $y \in \delta\left(\left(x^{2} y\right)\right)=y \delta\left(\left(x^{2}\right)\right)$. Suppose that $y \in y \delta\left(\left(x^{2}\right)\right)$. Then there exists $a \in \delta\left(\left(x^{2}\right)\right) \subseteq \delta(\operatorname{Jac}(R))=\operatorname{Jac}(R)$ such that $y=y a$. Which implies that $y(1-a)=0$. Since $1-a$ is unit, we have $y=0$, which is a contradiction. Thus we have, $x^{2} \in\left(x^{2} y\right)$. Then we can write $x^{2}=r x^{2} y$ for some $r \in R$. This implies that $x^{2}(1-r y)=0$. Since $1-r y$ is unit, we have $x^{2}=0$. Likewise, we get $y^{2}=0$. Now, choose another $z \in \operatorname{Jac}(R)$. Since $x y z \in(x y z)$ and $(x y z)$ is a 1-absorbing $\delta$-primary, we get $x y \in(x y z)$ or $z \in \delta((x y z))=z \delta((x y))$. First, assume that $x y \in(x y z)$. Then there exists $r \in R$ such that $x y=r x y z$, which implies that $x y(1-r z)=0$. Since $1-r z$ is unit, we have $x y=0$ which completes the proof. Now, assume that $x y \notin(x y z)$, that is, $z \in \delta((x y z))=$ $z \delta((x y))$. Then there exists $a \in \delta((x y)) \subseteq J a c(R)$ such that $z=z a$. This implies that $z(1-a)=0$ so that $z=0$. Now, choose $z=x+y$. Then by above argument, we have either $x y=0$ or $z=x+y=0$. If $z=x+y=0$, then we have $x=-y$ and so $x y=-y^{2}=0$ which completes the proof. Therefore, $\operatorname{Jac}(R)^{2}=(0)$.

Now, we will show that $R$ is a local ring. Choose maximal ideals $M_{1}, M_{2}$ of $R$. Now, put $I=M_{1} \cap M_{2}$. Since $M_{1}^{2} M_{2} \subseteq I$ and $I$ is a 1 -absorbing $\delta$ primary ideal, we have either $M_{1}^{2} \subseteq I \subseteq M_{2}$ or $M_{2} \subseteq \delta(I) \subseteq \delta\left(M_{1}\right)$. Case 1: Suppose that $M_{1}^{2} \subseteq M_{2}$. Since $M_{2}$ is prime, clearly we have $M_{1} \subseteq M_{2}$ which implies that $M_{1}=M_{2}$. Case 2: Suppose that $M_{2} \subseteq \delta\left(M_{1}\right)$. Since $\delta$ satisfies condition $(*), \delta\left(M_{1}\right)$ is proper. As $M_{1} \subseteq \delta\left(M_{1}\right)$ and $M_{1}$ is a maximal ideal, we have $M_{1}=\delta\left(M_{1}\right)$. Then we get $M_{2} \subseteq M_{1}$, which implies that $M_{1}=M_{2}$. Therefore, $R$ is a local ring.

(iii) $\Rightarrow\left(\right.$ i) Suppose that $R$ is a local ring with $\operatorname{Jac}(R)^{2}=(0)$. Let $I$ be a proper ideal of $R$ and $a b c \in I$ for some nonunits $a, b, c \in R$. Then $a, b, c \in \operatorname{Jac}(R)$ since $R$ is local. As $\operatorname{Jac}(R)^{2}=(0)$, we have $a b=0 \in I$. 
Therefore, $I$ is a 1-absorbing $\delta$-primary ideal of $R$.

It can be easily seen that, in Theorem $2.20,($ iii) always implies $(i)$ without any assumption on $\delta$. But we give some examples showing that the converse is not true if we drop the aforementioned assumptions on $\delta$.

Example 2.21. Let $R=\mathbb{Z}_{p^{3}}$, where $p$ is a prime number and $\delta(I)=R$ for every proper ideal I of $R$. Note that $\delta$ does not satisfy condition $(*)$ and note that every ideal $I$ of $R$ is 1 -absorbing $\delta$-primary. Thus $J a c(R)^{2} \neq(0)$, while $R$ is a local ring.

Example 2.22. Let $k$ be a field and consider the formal power series ring $R=k[[X]]$. Then $R$ is a local ring with unique maximal ideal $m=(X)$. Define expansion function $\delta$ as $\delta(I)=\sqrt{I}$ for every ideal $I$ of $R$. Then it is easy to see that every ideal of $R$ is a 1-absorbing $\delta$-primary ideal. Also, it is clear that $\delta$ satisfies condition $(*)$ and $\delta(\operatorname{Jac}(R))=\operatorname{Jac}(R)$ but not satisfy the condition $\delta(x I)=x \delta(I)$. Furthermore, $\operatorname{Jac}(R)^{2} \neq(0)$. Thus Theorem 2.20 fails without assumption $\delta(x I)=x \delta(I)$.

Corollary 2.23. Let $R$ be a ring. The following statements are equivalent.

(i) Every proper ideal is a 1-absorbing prime ideal of $R$.

(ii) Every proper principal ideal is a 1-absorbing prime ideal of $R$.

(iii) $R$ is local with $J a c(R)^{2}=(0)$.

Proof. $(i) \Leftrightarrow($ ii $)$ Follows from Proposition 2.19.

$($ ii $) \Rightarrow($ iii $)$ Let $\delta$ be the identity expansion function, that is, $\delta(I)=I$ for every ideal $I$ of $R$. Note that $\delta$ satisfies all axioms in Theorem 2.20. Then $R$ is a local ring with $\operatorname{Jac}(R)^{2}=(0)$.

$($ iii $) \Rightarrow($ i $)$ It is similar to Theorem $2.20($ iii $) \Rightarrow(i)$.

An ideal expansion $\delta$ is called a prime expansion if for any 1-absorbing primary ideal $I$ of $R, \delta(I)$ is a prime ideal of $R$.

Proposition 2.24. Let $R$ be a local ring with maximal ideal $M$ and $\delta$ be a prime expansion function of $\mathcal{J}(R)$. Assume that one of the following conditions holds:

(1) $\operatorname{Spec}(R)=\{\delta(0)\}$.

(2) $\operatorname{Spec}(R)=\{\delta(0), M\}$ and $\delta(0) M=0$.

Then every proper ideal of $R$ is 1-absorbing $\delta$-primary. 
Proof. Let $I$ be a proper ideal of $R$ and assume that (1) holds. Since $\delta(0)$ is maximal ideal and $\delta(I) \supseteq \delta(0)$, we conclude that $\delta(I)=R$ or $\delta(I)=\delta(0)$. Clearly $I$ is a 1-absorbing $\delta$-primary ideal of $R$. Now, assume that $\operatorname{Spec}(R)=$ $\{\delta(0), M\}$ and $\delta(0) M=0$. Since $\delta(0) \subseteq M$, we have $\sqrt{I}=M$ or $\sqrt{I}=$ $\delta(0)$. First assume that $\sqrt{I}=M$. Then $I$ is 1 -absorbing primary ideal so that $\delta(I)=M$ or $\delta(I)=\delta(0)$. If $\delta(I)=M$, we have then $I$ is 1-absorbing $\delta$ primary. So assume that $\delta(I)=\delta(0)$. Let $a b c \in I$ for some nonunit elements $a, b, c \in R$ such that $c \notin \delta(0)$. As $I \subseteq \delta(0)$, we get that either $a \in \delta(0)$ or $b \in \delta(0)$. Thus $a b=0 \in I$ which gives that $I$ is a 1 -absorbing $\delta$-primary ideal of $R$. In the remaining case, $I \subseteq \sqrt{I}=\delta(0) \subseteq \delta(I)$. A similar argument shows that $I$ is a 1 -absorbing $\delta$-primary ideal of $R$.

Let $f: R \rightarrow S$ be a ring homomorphism and $\delta, \gamma$ expansion functions of $\mathcal{J}(R)$ and $\mathcal{J}(S)$ respectively. Recall from [3] that $f$ is called a $\delta \gamma$-homomorphism if $\delta\left(f^{-1}(I)\right)=f^{-1}(\gamma(I))$ for each ideal $I$ of $S$. Also note that if $f$ is a $\delta \gamma$ epimorphism and $I$ is an ideal of $R$ containing $\operatorname{ker}(f)$, then $\gamma(f(I))=f(\delta(I))$.

Theorem 2.25. Let $f: R \rightarrow S$ be a ring $\delta \gamma$-homomorphism where $\delta, \gamma$ are expansion functions of $\mathcal{J}(R)$ and $\mathcal{J}(S)$ respectively. Suppose that $f(a)$ is nonunit in $S$ for every nonunit element a in $R$. Then the following statements hold.

(1) If $J$ is a 1-absorbing $\gamma$-primary ideal of $S$, then $f^{-1}(J)$ is a 1-absorbing $\delta$-primary ideal of $R$.

(2) If $f$ is an epimorphism and $I$ is a proper ideal of $R$ containing $\operatorname{ker}(f)$, then $I$ is a 1-absorbing $\delta$-primary ideal of $R$ if and only if $f(I)$ is a 1 -absorbing $\gamma$-primary ideal of $S$.

Proof. (1) Assume that $a b c \in f^{-1}(J)$, for some nonunit elements $a, b, c \in R$. Then $f(a) f(b) f(c) \in J$. Thus $f(a) f(b) \in J$ or $f(c) \in \gamma(J)$, which implies that $a b \in f^{-1}(J)$ or $c \in f^{-1}(\gamma(J))=\delta\left(f^{-1}(J)\right)$. Therefore, $f^{-1}(J)$ is a 1absorbing $\delta$-primary ideal of $R$.

(2) Suppose that $f(I)$ is 1-absorbing $\gamma$-primary ideal of $S$. Since $I=f^{-1}(f(I))$, we conclude that $I$ is a 1-absorbing $\delta$-primary ideal of $R$ by (1). Conversely, let $x, y, z$ be nonunit elements of $S$ with $x y z \in f(I)$. Then there exist $a, b, c \in R$ such that $x=f(a), y=f(b)$ and $z=f(c)$ with $f(a b c)=x y z \in f(I)$. Since $\operatorname{ker}(f) \subseteq I$, we then have $a b c \in I$. Since $I$ is a 1 -absorbing $\delta$-primary ideal of $R$ and $a b c \in I$, we conclude that $a b \in I$ or $c \in \delta(I)$ which gives that $x y \in f(I)$ or $z \in f(\delta(I))=\gamma(f(I))$. Thus $f(I)$ is a 1-absorbing $\delta$-primary ideal of $S$.

Let $\delta$ be an expansion function of $\mathcal{J}(\mathcal{R})$ and $I$ an ideal of $R$. Then the function $\bar{\delta}: \frac{R}{I} \longrightarrow \frac{R}{I}$ defined by $\bar{\delta}\left(\frac{J}{I}\right)=\frac{\delta(J)}{I}$ for all ideals $I \subseteq J$, becomes an expansion function of $\frac{R}{I}$. Then, we have the following result. 
Corollary 2.26. Let $R$ be a ring, $\delta$ an expansion function of $\mathcal{J}(\mathcal{R})$ and $I \subseteq J$ be proper ideals of $R$. Assume that $a+I$ is a nonunit element of $\frac{R}{I}$ for every nonunit element $a \in R$. Then $J$ is a 1-absorbing $\delta$-primary ideal of $R$ if and only if $\frac{J}{I}$ is a 1 -absorbing $\bar{\delta}$-primary ideal of $\frac{R}{I}$.

Proposition 2.27. Let $S$ be a multiplicatively closed subset of a ring $R$ and $\delta_{S}$ an expansion function of $\mathcal{J}\left(S^{-1} R\right)$ such that $\delta_{S}\left(S^{-1} I\right)=S^{-1}(\delta(I))$ for each ideal $I$ of $R$. If $I$ is a 1-absorbing $\delta$-primary ideal of $R$ such that $I \cap S=\varnothing$, then $S^{-1} I$ is a 1-absorbing $\delta_{S}$-primary ideal of $S^{-1} R$.

Proof. Let $I$ be a 1-absorbing $\delta$-primary ideal of $R$ such that $I \cap S=\varnothing$ and $\frac{a}{s} \frac{b}{t} \frac{c}{r} \in S^{-1} I$ for some nonunit elements $a, b, c \in R$ and $s, t, r \in S$ such that $\frac{a}{s} \frac{b}{t} \notin S^{-1} I$. Then $x a b c \in I$ for some $x \in S$. Since $I$ is a 1 -absorbing $\delta$-primary and $x a b \notin I$, we conclude that $c \in \delta(I)$. Thus $\frac{c}{r} \in S^{-1}(\delta(I))=\delta_{S}\left(S^{-1} I\right)$ which completes the proof.

Let $S$ be a multiplicatively closed subset of a ring $R$ and $I$ an ideal of $R$. The next example shows that if $S^{-1} I$ is a 1-absorbing $\delta_{S}$-primary ideal of $S^{-1} R$, then $I$ need not to be a 1-absorbing $\delta$-primary ideal of $R$.

Example 2.28. Let $p \neq q$ be two prime numbers. Set $I=p q \mathbb{Z}$ and $\delta$ be an ideal expansion such that $\delta(I)=I+q \mathbb{Z}$ for each ideal $I$ of $\mathbb{Z}$. Clearly, $I$ is not a 1-absorbing $\delta$-primary ideal of $\mathbb{Z}$ as it is shown in Example 2.5. Now, let $S=\mathbb{Z} \backslash p \mathbb{Z}$ and note that $S^{-1} I=S^{-1}(p \mathbb{Z})$. Let $\frac{a}{r_{1}} \frac{b}{r_{2}} \frac{c}{r_{3}} \in S^{-1} I$ for some nonunit elements $\frac{a}{r_{1}}, \frac{b}{r_{2}}, \frac{c}{r_{3}} \in S^{-1} \mathbb{Z}$. Note that $\frac{x}{r} \in S^{-1} \mathbb{Z}$ is nonunit if and only if $x \in p \mathbb{Z}$. Thus $a \in p \mathbb{Z}$ and $b \in p \mathbb{Z}$. Which gives that $\frac{a}{r_{1}} \frac{b}{r_{2}} \in S^{-1} I$ and hence $S^{-1} I$ is a 1 -absorbing $\delta_{S}$-primary ideal.

Let $R_{1}$ and $R_{2}$ be two rings, let $\delta_{i}$ be an expansion function of $\mathcal{J}\left(\mathcal{R}_{i}\right)$ for each $i \in\{1,2\}$ and $R=R_{1} \times R_{2}$. For a proper ideal $I_{1} \times I_{2}$, the function $\delta_{\times}$ defined by $\delta_{\times}\left(I_{1} \times I_{2}\right)=\delta_{1}\left(I_{1}\right) \times \delta_{2}\left(I_{2}\right)$ is an expansion function of $\mathcal{J}(\mathcal{R})$. The following result characterizes the 1 -absorbing $\delta$-primary ideals of the direct product of rings.

Theorem 2.29. Let $R_{1}$ and $R_{2}$ be rings, $R=R_{1} \times R_{2}$ and let $\delta_{i}$ be an expansion function of $\mathcal{J}\left(\mathcal{R}_{i}\right)$ for $i=1,2$. Then the following statements are equivalent:

(1) I is a 1-absorbing $\delta_{\times}$-primary ideal of $R$.

(2) $I$ is a $\delta_{\times}$-primary ideal of $R$.

(3) Either $I=I_{1} \times R_{2}$, where $I_{1}$ is a $\delta_{1}$-primary ideal of $R_{1}$ or $I=R_{1} \times I_{2}$, where $I_{2}$ is a $\delta_{2}$-primary ideal of $R_{2}$ or $I=I_{1} \times I_{2}$, where $I_{1}$ and $I_{2}$ are proper ideals of $R_{1}, R_{2}$, respectively with $\delta_{1}\left(I_{1}\right)=R_{1}$ and $\delta_{2}\left(I_{2}\right)=R_{2}$. 
Proof. (1) $\Leftrightarrow(2)$. This follows from Theorem 2.6.

$(2) \Leftrightarrow(3)$ Let $I$ be a $\delta_{\times}$-primary ideal of $R$. Hence $I$ has the form $I=I_{1} \times I_{2}$ where $I_{1}$ and $I_{2}$ are ideals of $R_{1}$ and $R_{2}$ respectively. Without loss of generality, we may assume that $I=I_{1} \times R_{2}$ for some proper ideal $I_{1}$ of $R_{1}$. We show that $I_{1}$ is a $\delta_{1}$-primary ideal of $R_{1}$. Deny. Then there are $a, b \in R_{1}$ such that $a b \in I_{1}, a \notin I_{1}$ and $b \notin \delta_{1}\left(I_{1}\right)$. Hence $(a, 1)(b, 1) \in I_{1} \times R_{2}$. Which implies that $(a, 1) \in I_{1} \times R_{2}$ or $(b, 1) \in \delta_{\times}\left(I_{1} \times R_{2}\right)$ and so $a \in I_{1}$ or $b \in \delta_{1}\left(I_{1}\right)$, which gives a contradiction. Now suppose that both $I_{1}$ and $I_{2}$ are proper. As $(1,0)(0,1) \in I_{1} \times I_{2}$ and $(1,0),(0,1) \notin I_{1} \times I_{2}$, we have $(1,0),(0,1) \in$ $\delta_{\times}\left(I_{1} \times I_{2}\right)=\delta_{1}\left(I_{1}\right) \times \delta_{2}\left(I_{2}\right)$. Therefore $\delta_{1}\left(I_{1}\right)=R_{1}$ and $\delta_{2}\left(I_{2}\right)=R_{2}$. The converse is clear.

The following example proves that the condition " $\delta\left(I_{i}\right)=P$ for all $i \in$ $\{1,2, \ldots, n\} "$ is necessary in Proposition 2.18 .

Example 2.30. Let $R=R_{1} \times R_{2}$ where $R_{1}=R_{2}=\mathbb{Z}$ and $\delta_{1}=\delta_{2}=\delta$ where $\delta(I)=\sqrt{I}+2 \mathbb{Z}$ for every ideal $I$ of $\mathbb{Z}$. Consider the ideals $K=4 \mathbb{Z} \times \mathbb{Z}$ and $L=\mathbb{Z} \times 9 \mathbb{Z}$ of $R$. Thus $\delta_{\times}(K)=\delta_{1}(4 \mathbb{Z}) \times \delta_{2}(\mathbb{Z})=2 \mathbb{Z} \times \mathbb{Z}$ and $\delta_{\times}(L)=$ $\delta_{1}(\mathbb{Z}) \times \delta_{2}(9 \mathbb{Z})=\mathbb{Z} \times \mathbb{Z}$. Moreover, $K$ and $L$ are 1 -absorbing $\delta_{\times}$-primary ideal. But $K \cap L=4 \mathbb{Z} \times 9 \mathbb{Z}$ is not a 1-absorbing $\delta_{\times}$-primary ideal by Theorem 2.29.

In view of Theorem 2.29, we have the following result.

Theorem 2.31. Let $R_{1}, R_{2}, \ldots, R_{n}$ be commutative rings with nonzero identity and $R=R_{1} \times R_{2} \times \cdots \times R_{n}$ where $n \geq 2$. Let $\delta_{i}$ be an expansion function of $\mathcal{J}\left(\mathcal{R}_{i}\right)$ for each $i=1,2, \ldots, n$. Then the following statements are equivalent.

(1) $I$ is a 1-absorbing $\delta_{x}$-primary ideal of $R$.

(2) $I=I_{1} \times I_{2} \times \cdots \times I_{n}$ and either for some $k \in\{1,2, \ldots, n\}$ such that $I_{k}$ is a 1-absorbing $\delta_{k}$-primary ideal of $R_{k}$ and $I_{j}=R_{j}$ for each $j \in$ $\{1,2, \ldots, n\} \backslash\{k\}$ or $I_{\alpha_{i}}$ 's are proper ideals of $R_{\alpha_{i}}$ for $\left\{\alpha_{1}, \alpha_{2}, \ldots, \alpha_{k}\right\} \subseteq$ $\{1,2, \ldots, n\}$ and $\left|\left\{\alpha_{1}, \alpha_{2}, \ldots, \alpha_{k}\right\}\right| \geq 2$ with $\delta_{\alpha_{i}}\left(I_{\alpha_{i}}\right)=R_{\alpha_{i}}$, and $I_{j}=R_{j}$ for all $j \in\{1,2, \ldots, n\} \backslash\left\{\alpha_{1}, \alpha_{2}, \ldots, \alpha_{k}\right\}$.

Proof. It can be obtained by using mathematical induction on $n$.

Let $A$ be a ring and $E$ an $A$-module. Then $A \ltimes E$, the trivial (ring) extension of $A$ by $E$, is the ring whose additive structure is that of the external direct sum $A \oplus E$ and whose multiplication is defined by $(a, e)(b, f):=(a b, a f+$ be) for all $a, b \in A$ and all $e, f \in E$. (This construction is also known by other terminology and other notation, such as the idealization $A(+) E$.) The basic properties of trivial ring extensions are summarized in the books [10], [9]. Trivial ring extensions have been studied or generalized extensively, often because of their usefulness in constructing new classes of examples of rings satisfying various properties (cf. $[1,6,7,11]$ ). In addition, for an ideal $I$ of 
$A$ and a submodule $F$ of $E, I \ltimes F$ is an ideal of $A \ltimes E$ if and only if $I E \subseteq F$. Moreover, for an expansion function $\delta$ of $A$, it is clear that $\delta_{\ltimes}$ defined as $\delta_{\ltimes}(I \ltimes F)=\delta(I) \ltimes E$ is an expansion function of $A \ltimes E$. Also as usual, if $c \in A$ then $(F: c)=\{e \in E \mid c e \in F\}$.

Theorem 2.32. Let $A$ be a ring, $E$ an $A$-module and $\delta$ be an expansion function of $\mathcal{J}(A)$. Let $I$ be an ideal of $A$ and $F$ a submodule of $E$ such that $I E \subseteq F$. Then the following statement hold:

(1) If $I \ltimes F$ is a 1-absorbing $\delta_{\ltimes}$-primary ideal of $A \ltimes E$, then $I$ is a 1 absorbing $\delta$-primary ideal of $A$.

(2) Assume that $(F: c)=F$ for every $c \in A \backslash I$. Then $I \ltimes F$ is a 1 -absorbing $\delta_{\ltimes}$-primary ideal of $A \ltimes E$ if and only if $I$ is a 1-absorbing $\delta$-primary ideal of $A$.

Proof. (1) Assume that $I \ltimes F$ is a 1-absorbing $\delta_{\ltimes}$-primary ideal of $A \ltimes E$ and let $a, b, c$ be nonunit elements of $A$ such that $a b c \in I$. Thus $(a, 0)(b, 0)(c, 0)=$ $(a b c, 0) \in I \ltimes F$ which implies that $(a, 0)(b, 0) \in I \ltimes F$ or $(c, 0) \in \delta_{\ltimes}(I \ltimes F)=$ $\delta(I) \ltimes E$. Therefore $a b \in I$ or $c \in \delta(I)$ and so (1) holds.

(2) By (1), it suffices to prove the "if" assertion. Let $(a, s),(b, t),(c, r)$ be nonunit elements of $A \ltimes E$ such that $(a, s)(b, t)(c, r)=(a b c, b c s+a c t+a b r) \in$ $I \ltimes F$. Clearly, $a b c \in I$ and so $a b \in I$ or $c \in \delta(I)$ since $I$ is a 1-absorbing $\delta$-primary ideal of $A$. If $c \in \delta(I)$, then $(c, r) \in \delta(I) \ltimes E=\delta_{\ltimes}(I \ltimes F)$. Hence, we may assume that $c \notin \delta(I)$. Then $a b \in I$. As bcs $+a c t+a b r \in F$ and $a b r \in F$, we get that $b c s+a c t \in F$. This implies $b s+a t \in(F: c)=F$ and so $(a, s)(b, t)=(a b, a t+b s) \in I \ltimes F$. Therefore $I \ltimes F$ is a 1-absorbing $\delta_{\ltimes}$-primary ideal of $A \ltimes E$.

Corollary 2.33. Let $A$ be a ring, $E$ an $A$-module and $\delta$ be an expansion function of $\mathcal{J}(A)$. Let $I$ be a proper ideal of $A$. Then $I \ltimes E$ is a 1-absorbing $\delta_{\ltimes}$-primary ideal of $A \ltimes E$ if and only if $I$ is a 1-absorbing $\delta$-primary ideal of A.

Acknowledgement. The authors would like to thank the referees for their valuable comments which helped to improve the manuscript.

\section{References}

[1] Anderson, D. D. and Winders, M., Idealization of a module. J. Comm. Alg., 1(1) (2009), 3-56.

[2] Badawi, A., On 2-absorbing ideals of commutative rings. Bulletin of the Australian Mathematical Society, 75(3) (2007), 417-429. 
[3] Badawi, A. and Fahid, B., On weakly 2-absorbin $\delta$-primary ideals of commutative ring. Georgian. Math. J., 27(4) (2017), 503-516.

[4] Badawi, A. and Celikel, E. Y., On 1-absorbing primary ideals of a commutative rings. J. Algebra Appl., (2020), 2050111.

[5] Badawi, A., Sonmez, D., Yesilot, G., On weakly $\delta$-semiprimary ideals of commutative rings. Algebra Colloq., 25 (2018), 387-398.

[6] Dobbs, D. E., Elkhalfi, A., Mahdou, N., Trivial extensions satisfying certain valuation-like properties, Comm. Algebra, 47(5) (2019), 2060-2077.

[7] Dumitrescu, T., Mahdou, N., Zahir, Y., Radical factorization for trivial extensions and amalgamated duplication rings. Journal of Algebra and Its Applications, 20(02) (2021), 2150025.

[8] Fahid, B. and Dongsheng, Z., 2-absorbing $\delta$-primary ideals in commutative rings. Kyungpook Mathematical Journal, 57 (2) (2017), 193-198.

[9] Glaz, S., Commutative Coherent Rings. Lecture Notes in Math. 1371, Springer-Verlag, Berlin, 1989.

[10] Huckaba, J. A., Commutative Rings with Zero Divisors. Dekker, New York, 1988.

[11] Kabbaj, S. and Mahdou, N., Trivial extensions defined by coherent-like conditions. Comm. Algebra, 32 (10) (2004), 3937-3953.

[12] Kaplansky, I., Commutative Rings. rev. ed., Univ. Chicago Press, Chicago, 1974.

[13] Ulucak, G., Tekir, Ü., Koc, S., On $n$-absorbing $\delta$-primary ideals. Turkish Journal of Mathematics, 42 (4) (2018), 1833-1844.

[14] Yassine, A., Nikmehr, M. J., Nikandish, R., On 1-absorbing prime ideals of commutative rings. J. Algebra Appl., (2020), 2150175.

[15] Zhao, D., $\delta$-primary ideals of commutative rings. Kyungpook Math. J., 41 (1) (2001), 17-22 
Abdelhaq El Khalfi,

Laboratory of Modelling and Mathematical Structures

Department of Mathematic, Faculty of Science and Technology of Fez, Box

2202, University S.M. Ben Abdellah Fez, Morocco.

Email: abdelhaq.elkhalfi@usmba.ac.ma

Najib Mahdou,

Laboratory of Modelling and Mathematical Structures

Department of Mathematics, Faculty of Science and Technology of Fez, Box

2202, University S.M. Ben Abdellah Fez, Morocco.

Email: mahdou@hotmail.com

Ünsal Tekir,

Department of Mathematics,

Marmara University,

Istanbul, Turkey.

Email: utekir@marmara.edu.tr

Suat Koç,

Department of Mathematics,

Marmara University,

Istanbul, Turkey.

Email: suat.koc@marmara.edu.tr 